\title{
Application of Wearable Technology in Education
}

\author{
Ximei Qu${ }^{1}$, Jiahuan Wang2 ${ }^{2}$ Rong Miao ${ }^{3 *}$ \\ ${ }^{1}$ Department of Information, Zhejiang Open University, Hangzhou, China \\ ${ }^{2}$ Vocational School, Zhejiang Open University, Hangzhou, China \\ ${ }^{3}$ Graduate School of Education, Peking University, Beijing, China \\ Email: natasha210@126.com, ${ }^{\star}$ miao@gse.pku.edu.cn
}

How to cite this paper: Qu, X.M., Wang, J.H. and Miao, R. (2021) Application of Wearable Technology in Education. Open Access Library Journal, 8: e7630. https://doi.org/10.4236/oalib.1107630

Received: June 10, 2021

Accepted: November 12, 2021

Published: November 15, 2021

Copyright (C) 2021 by author(s) and Open Access Library Inc.

This work is licensed under the Creative Commons Attribution International License (CC BY 4.0).

http://creativecommons.org/licenses/by/4.0/

\begin{abstract}
Wearable technology is very popular in recent years. Since the advent of Glass Google, lots of kinds of wearable devices have been developed. There is more and more wearable technology in education. Through the literature and field survey, the researchers conducted studies on wearable technology and its application in education. We find that the scope of application involves many fields, such as medical, entertainment, health, military, education, and so on. The effective use of wearable technology in education needs to improve the devices to meet the needs of education.
\end{abstract}

\section{Subject Areas}

Information Science

\section{Keywords}

Wearable Technology, Wearable Device, Application

\section{Introduction}

In recent years, with the development of mobile Internet and hardware technology, the function of wearable devices has been improved. It becomes more convenient, easier to interact and it has better real-time performance. Wearable systems range from micro sensors seamlessly integrated into textiles through consumer electronics embedded in fashionable clothes and computerized watches to belt worn PCs with a head mounted display [1]. They have been used wider in many fields, such as medical treatment, fire protection, military, disabled aid, entertainment, design, etc. Wearable devices also lead to the expectation that people will apply them to teaching and learning with their unique advantages. 
However, the security problems in physical activities have become one of the main factors that restrict the policy of physical education in primary and secondary schools in China [2]. Using wearable devices may be a solution. In this paper, we will talk more about wearable devices in education.

\section{Literature Review}

\subsection{Definition}

The terms "wearable technology", "wearable devices", and "wearables" all refer to electronic technologies or devices that are incorporated into items of clothing and accessories which can comfortably be worn on the body. These wearable devices can perform many of the same computing tasks as mobile phones and laptop computers; however, in some cases, wearable technology can outperform these hand-held devices entirely. Wearable technology tends to be more sophisticated than hand-held technology on the market today because it can provide sensory and scanning features not typically seen in mobile and laptop devices, such as biofeedback and tracking of physiological function [3].

In the Horizon Report of 2016 K-12 Edition, wearable technology refers to smart devices that can be worn by users, taking the form of an accessory such as jewelry or eyewear. Smart textiles also allow items of clothing such as shoes or jackets to interact with other devices. The wearable format enables the convenient integration of tools into users' everyday lives, allowing seamless tracking of personal data such as sleep, movement, location, and social media interactions [4].

Described as embeddable technology that is implanted underneath the skin, this category gives a whole new meaning to the term "wearable". An electronic engineer, for example, has designed a GPS-enabled shoulder implant called Southpaw that will prevent people from getting lost during outdoor expeditions. Another engineer has pioneered magnets that can be embedded into users' ears allowing them to listen to audio at any time [5].

Compared with the traditional electronic equipment, wearable devices have the advantages of being convenient to carry and good interact, etc., which can be used as information communication tools, seamlessly existing in people's lives, without distracting the user's life and work attention. Users can sense the environment and control the device anytime, anywhere.

When dealing with daily affairs, Google glasses can provide users with relevant information. Users can get involved on the internet with voice commands to reply e-mails and to do much other work. The glasses can also provide users with relevant warning information. For example, if the train that users take to campus is late, Google glasses can let them know and provide an alternative route.

Jawbone's UP wristband connects with apps that display information about how many steps the wearer has taken, heart rate, sleeping and other healthrelated information, along with providing tailored recommendations for exercise 
and nutrition.

In this chapter, we do not distinguish between "wearable technology" and "wearable devices". Although the former emphasizes the technology, and the latter emphasizes the devices, but the wearable device uses wearable technology. Moreover, now we often use wearables to refer to "wearable devices" or wearable technology.

According to the above definition, we believe that wearable devices are smart devices (such as jewelry, glasses and watches and other accessories) that can be worn or embedded in the human body. These devices can integrate multimedia, wireless communications, flexible screen, GPS, micro-sensing, virtual reality, biometric and other cutting-edge technology, while they will be related to the human body to collect, process, share information and provide feedback anytime, anywhere by the large data platform, intelligent cloud and mobile Internet.

\subsection{Research Progress}

Wearable technology is not a new category; one of the most popular early incarnations of the wearable technology was HP's calculator watch, which was introduced in the 1980s. In 2010, Nike+ Sportband was introduced as a device that can communicate with the sensor hidden in the shoes to tell people the details about running. In 2012, the major companies in international consumer electronics sector display their wearable devices at trade shows. It was in 2013, however, that the introduction of Google Project Glass that has opened the era of wearable technology.

"The wearable technology will drive innovation just like the personal computers of the 1980s and the current mobile computer tablets," said Mary Meeker, called the Internet Queen. "Some people have laughed at the wearable technology, such as Google's glasses, and it is just like that some people laugh at personal computers and internet [6]." Wearable devices have not been as widely accepted as today's smart phones, but in the entertainment, health care, security and other fields, they have begun to play their unique role.

Using the "wearable device" as a keyword, we search in the Web of Science database. From the macro perspective, some studies demonstrate the design, implementation and prospects of wearable devices. Focusing on the use of wearable equipment in a particular area, there are medical, sports, fitness and education applications.

Nowadays, wearable devices have been used in the field of infotainment, such as Apple's Apple watch, Google's Google glass, Sony's Smart watch, etc., and they are launched by major electronics companies. These devices mainly meet the needs of information communication and people's entertainment [7].

The current wearable technology is also widely used in the field of security protection. Using built-in chip technology to detect and track the user's geographical location, and the data transmitted to the terminal equipment, the wearable devices can prevent the user from being lost to ensure users' security 
outdoor. At present, the wearable devices mainly meet the needs of the elderly and children to prevent them from being lost. For example, GTX of United States and the Aetrex shoe company jointly developed positioning shoes, which are embedded GPS chip, especially for patients with Alzheimer's disease.

Wearable technology has a very broad prospect for development in the medical health field. Today, these wearable devices such as smart bracelets, watches and collars can help healthcare professionals detect data such as blood sugar, heart rate and exercise status so that they can keep track of metrics and assign a health management program to users to protect sudden changes in the body caused by sudden disease. Wearable devices will bring a revolution for the medical equipment industry [8]. Google Glass is also thought to bea good way for trainees to easily acquire intraoperative footage for self-review [9]. The medical devices in the future will be more and more miniature, and be worn and even embedded within the human body.

In sports and training field, the United States company Zepp has developed wearable devices that are suitable for and can be used in the baseball, golf and tennis. Athletes wear a lightweight motion sensor, which can capture their movements, and stream sports data to the data server (or mobile client) wirelessly. Athletes and coaches can analyze and play back the actions by looking at the corresponding data to improve their performance. Spelmezan and others have proposed a wearable system for skiing that can identify errors often made by beginners, such as uneven distribution of boots, erroneous postures, incorrect twist angles of upper body, and bending of knees. Once the error posture or action is detected, the wearable devices will provide automatic feedback to the mobile host device, the coach can remotely guide their posture and action. Alahakone and others proposed a gait research and recovery method. When people do exercise in the treadmill, wearable devices can identify the heelstrike and heeloff through the inertial sensor technology, achieving the effect of scientific gait training [10].

There are also some studies aiming at specific people, such as the blind and the elderly. And a large part of these papers are conference papers. There are some empirical studies.

Sumin Koo and Fallon [11] have studied what dimensions consumers prefer to track using wearable technology to achieve a healthier lifestyle and how these tracking dimensions are related, by the way of online survey. The enlightenment of the paper is that designers are encouraged to make wearable technology products that are durable, easy to care for, attractive in design, comfortable to wear and use, able to track preferred dimensions, appropriate for various consumers, unobtrusive, portable, and small. The research will guide wearable technology and fashion industry professionals in the development process of wearable technology to benefit consumers by helping them being more self-aware, empowering them to develop a healthier lifestyle, and ultimately increasing their quality of life and well-being. 
Belsi, Papi and McGregor [12] designed a qualitative study using focus groups with patients with osteoarthritis. Patients' responses suggested a positive attitude on the impact wearable technology could have on the management of osteoarthritis. It was perceived that the use of wearable devices would benefit patients in terms of feeling in control of their condition, providing them with awareness of their progress, empowering in terms of self-management and improving communication with their clinician. The information obtained from this study suggests that introducing wearable technology into patient-centered care could enhance patient experience in the field of osteoarthritis and beyond.

\section{Cases}

Currently on the market, the mainstream wearable devices include smart glasses with Google glasses as the market leader, smart watches such as Samsung, Sony, Pebble, as well as smart bracelet of Fitbit, Jawbone and Nike coming out on top. There are also virtual reality helmets, such as Oculus Rift, Project Morpheus of Sony, which are developed primarily for games that can provide immersive, more innovative gaming experiences for users.

The following summary introduces some typical application cases of wearable technology.

\subsection{Applications in K-12 PE Classes}

Wearable technology can be applied in K-12 physical education classes and help students to establish health habits and help teachers adjust exercise intensity and density according to the information such as heart rate displayed on apps.

The following case comes from the seventh primary school of Zhaoqing City, Guangdong Province, and the teacher is Zhao. This class is called "durable run-campus orienteering" In the physical education class.

This course takes full advantage of the full color LED display, tablet computer, smart bracelet, orienteering marking device and other equipment. Tablet computer, smart bracelet and orienteering marking device can help collect students' data and upload them to the teacher side in real time, and then do statistical analysis. Then students' information can be displayed through the stage LED. Students can see their own real-time movement, and teachers at any time can get to know the movement of students and grasp the situation.

The order of class contents is: classroom routine $->$ classroom introduction (the method of Flipped Classroom) -> jogging + game, warm up -> campus orienteering exercise.

The teacher uses a variety of wearable devices, such as smart wristbands, orienteering marking device, in order to achieve the training requirements for students in this class:

1) Campus orienteering exercise

- According to the problems of preview and courses' key and difficult points, the teacher explains the professional actions and precautions of orienteering. 
Then, the teacher sends the first map to the tablets of each student through Network disk.

- Students plan the personalized map that owned their group on the paper maps, and upload the pictures they take to the tablets.

- Students start the orienteering exercise under the two maps. Students test the physical data as soon as finishing the orienteering.

- After finishing the test, showing the students' exercise load and trajectory in some parts of groups.

2) Rules of campus orienteering

- Getting four-student crews (each group has two tablets, two sports bracelets, two orienteering machines).

- Students must have two different routes of campus orienteering race according to the two maps offered by tablets.

- The personalized maps that students planned by themselves can't be the same as the routes that teachers hand out.

- Each group must record the sports time through the orienteering machine and routes by scanning QR codes.

- Students must then complete the corresponding exercise once they arrive at every site.

In this lesson, wearable devices greatly help teachers and students understand students' changes in heart rate, as well as the moving trajectory in the process. It plays a great help for the teacher's class reflection, adjustment of physical exercise. At present, the school is still communicating with the relevant enterprises, expecting enterprises to develop wearable devices and supporting platform more suitable for physical education class, to ensure that the wearable devices can provide in-time feedback about the students' data about heart rate, exercise load. What is more, the wearable devices can make real-time statistics, analysis and provide better feedback. The devices that are currently used are not perfect for these purposes.

\subsection{Oculus Rift-A Virtual Reality Headset}

We usually think that three main sources of power to promote the rise of the Internet is three Gs: the Game, the Gamble, and the Girl. Even now, three Gs is also the traffic sources and benefit sources of many Internet giants, largely because they represent a standard for both basic and long-term human impulse. Historically, games also change with the transition of platforms.

Oculus Rift, designed for electronic games at first, can provide virtual reality experience through goggles. You Visit has adapted over 1000 virtual college tours so they can be viewed on Oculus Rift headsets. In 2014, Stony Brook University in New York and University of New Haven in Connecticut, for example, planned to implement this wearable technology into their marketing efforts. Virtual tours would allow students to go into campus spaces not typically open to visitors. From 2011 to 2014, the You Visit tour of Yale has been viewed more 
than 240,000 times, with an average of nearly 10 minutes spent per visit [13]. The Oculus Rift headset is also enabling students to explore potentially dangerous situations from the safety of the classroom. One virtual education expert has created a virtual construction worksite where engineering students can identify unsafe areas without exposure to harm. Health care research and training continues to advance the potential of wearable technology, as well. Rift has been used to help treat patients suffering from 'Phantom Limb' syndrome. Studies have shown that these patients can better come to terms with their loss and adapt their brains accordingly by seeing a virtual recreation of that limb. Oculus Rift can also help escape the stresses of modern life [14]. The Medical Virtual Reality group at the University of Southern California has developed simulations for wearable technology use for clinical purposes [15]. One of their projects focuses on medical training under simulated battlefield conditions.

\subsection{Cellphone-Charging Shirt}

Researchers at the University of South Carolina converted the fibers of a t-shirt into activated carbon, turning into a wearable hybrid super-capacitator that can charge portable electronic devices. The inventors claim that the process they used on the $\mathrm{t}$-shirt is less expensive, and greener, in comparison to conventional methods of creating electric storage devices.

\section{Discussion and Conclusion}

The development of wearable technology has just started. In the future, wearable products will grow at a faster rate. According to Tractica's forecast, Apple Watch in the next few years will continue to maintain the leader position in the wearable field, wearable products will focus on the field of health care; and mobile phones will become a big control platform.

In 2013, the National Development and Reform Commission of China issued a notice supporting the development and industrialization of wearable devices, which can provide a good support in policy. From the marketing point of view, on the one hand, with the popularity of the Internet and the improvement of consumer power, consumers' buying willingness and purchasing power are increasing. On the other hand, with the growing population aging and the improvement of education, wearable technology has been expected to gradually expand from simple exercise to health care, safe positioning and entertainment experience and other aspects. These are the basis for the development of wearable devices, but at the same time, its development is also facing a lot of problems and challenges, there exist the problems of battery and relying on mobile phones in smart watches.

As we all know, Apple has popularized existing technologies for four times: with the Macintosh computer in 1984, the iPod in 2001, the iPhone in 2007 and the iPad in 2010. Recently, the faithful have prayed that Apple will pull it off again with its smart watch. The smart watch can display many of the apps that 
are popular on smart phones, without hassle of having to pull out a phone. So far, however, the category has remained a niche plaything for geeks and athletes. The smart watches in other firms have the same situation, such as Moto 360, Motorola's smart watches most can only make calls or perform other functions if an accompanying phone is nearby.

What's more, Apple's smart phone's battery lasts for 18 hours before it needs more juice from a magnetic charger. The Apple Watch also needs to be close to an iPhone in order to function, which detracts from its usefulness.

At present, wearable devices on the market cannot have a stable consumer group. According to statistics, taking smart bracelet for an example, $42 \%$ of users no longer use the product, and nearly half of the users quit in a month, and 90\% of users quit in 3 months [16]. Most of the using habits are still formatting, and the user's dependence on the product is still weak. We think that excellent interactive design is likely to promote the user to take the initiative to adapt to new forms of interaction and products, promoting the user to pay for the better interactive experience, while enhancing the irreplaceability of the product, which is conducive to helping to form a stable consumer group.

Meanwhile, wearable devices have so far lacked the elegant design. Even the fashion models who were hired to strut around demonstrating Google Glass struggled to make it look stylish. Apple has hired fashion-conscious executives from luxury brands like Burberry and YSL to make its watch attractive, but it is not yet obvious that it has cracked the cool code.

Maybe, for smart watches and other wearable devices, to become mainstream products will take some time. We think, maybe in some day, the advances in technology will help to solve the problem of battery and the reliance on being close to an iPhone.

Nevertheless, the heat of wearable technology is still continuing. We think that the next few trends are (especially about education):

1) Application in education is developing well

Students in Minnesota's Westonka School Distinct use the Heart Zones System, a tracker that measures their heart rate, speed, distance, and more. The distinct adopted the system to combat a drop in PE enrollment and promote fitness; educators have found that the wearable devices help students develop motivation and have increased engagement [17].

Schools are also introducing wearable devices into physical education classes to personalize the curriculum through real-time feedback and grades based on individual skill mastery.

In China, we have consulted the school teachers in primary and secondary schools, and they think that real-time feedback in the wearable devices is very important. There are already many products that can be used to analyze the heart rate, movement trajectory and sports load of the students after PE class, but this cannot help the teachers in the classroom to adjust the exercise intensity in time. If there are suitable wearable devices with a matching qualified system 
for PE classes, which can provide timely feedback of information about sports load, heart rate and so on, they will be greatly welcomed.

Except for application in physical education classes, another compelling use of wearable technology is its potential to enhance fieldtrips and fieldwork. For instance, wearable cameras such as the Kickstarter-funded, GPS-enabled Memoto can instantly capture hundreds of photographs of data about a user's surroundings on a class trip to a museum or an offsite geology dig that can be later accessed via email or other online application [18]. The Contour Video Camera is another such device, currently favored by extreme athletes, that records and streams HD video. There is an increasing demand from users for all of their special moments to be seamlessly captured, but it is becoming less desirable to have to carry cumbersome devices. As technologies are continuously designed to be smaller and more mobile, wearable devices are a natural progression in the revolution of technology.

What's more, wearable devices can help students get rid of danger. When students are working in the lab, smart jewelry, clip-on earrings or other accessories can be used to alert the dangerous situation. So far, these types of devices are mostly being developed in university labs, including "ExposureTrack"designed at Arizona State University in collaboration with inXol that alerts people of working conditions that could potentially endanger their health [19]. One virtual education expert has created a virtual construction worksite where engineering students can identify unsafe areas without exposure to harm by Oculus Rift. Health care research and training continue to advance the potential of wearable technology, as well. The Medical Virtual Reality group at the University of Southern California has developed simulations for wearable technology use for clinical purposes [15]. One of their projects focuses on medical training under simulated battlefield conditions.

2) Operating system of wearables is recently concerned

Google Glass has limited success, and Google is now focusing on providing the operating system of choice for smart watches. In wearables, it is likely that companies will make a fortune from the operating system than from selling the hardware. Many developers will wait and see which operating system becomes dominant before invest money, time and effort in wearables. Apple and Google are going head-to-head to develop the operating system, which will unite different areas of people's lives, from their watches and phones to their cars and home appliances.

Thanks largely to the smart phone boom, chips and sensors have become cheaper and smaller. This has helped wearables move "from 'Star-Trek'-like dream to reality". But it is still some years before their full potential starts to be realized.

3) Less, not more

As mentioned earlier, the smart watches must be close to the smart phones, and many features are the same as the phone app. However, maybe someday 
wearable technology will provide us with a "persistent" digital identity, melding the functions of house key, credit card and a driving license in one small gadget worn on the wrist or neck, which is a little like a wristband (called a MagicBand) to get on rides, pay for food and enter hotel rooms in the Disney World theme park in Orlando.

Wearable devices hold the potential to transform some industries. Clinical trials could become cheaper and more accurate if drug makers give wearable monitors to the patients taking part. Hospitals and doctors' surgeries could use such monitors to reduce the need for home visits. Insurance firms could enter a new age in which they reduce risk as well as provide cover for it. One American health insurer is already handing out health-monitoring bands to customers, promising lower premiums for those who exercise more. Banks could reward customers who use the identity-verifying features of wearables, to cut the risk of card fraud. In PE classes, wearable technology can also help students and teachers adjust exercise intensity.

In short, wearable devices can not only help us better understand our own body, but also help us better explore the outside world, and even the universe. It is difficult, but the opportunities are boundless.

\section{Funding}

Science and Technology Innovation Plan for College Students in ZheJiang Province in 2020 (ZX2002052002); research on the Construction of the First Batch of Provincial Curriculum Ideological and Political Demonstration Project "Introduction to Artificial Intelligence" in Zhejiang Province.

\section{Conflicts of Interest}

The authors declare no conflicts of interest.

\section{References}

[1] Lukowicz, P., Kirstein, T. and Tröster, G. (2004) Wearable Systems for Health Care Applications. Methods of Information in Medicine, 43, 232-238. https://doi.org/10.1055/s-0038-1633863

[2] Sun, C. (2011) Study on Exercise Safety Problems and Countermeasures in Primary and Secondary Schools. Sports Expo, 13, 63.

[3] Tehrani, K. and Andrew, M. (2014) Wearable Technology and Wearable Devices: Everything You Need to Know. Wearable Devices Magazine.

[4] Adams Becker, S., Freeman, A., Giesinger Hall, C., Cummins, M. and Yuhnke, B. (2016) NMC/CoSN Horizon Report: 2016 K-12 Edition. The New Media Consortium, Austin.

[5] Johnson, L., Adams Becker, S., Estrada, V. and Freeman, A. (2014) NMC Horizon Report: 2014 K-12 Edition. The New Media Consortium, Austin.

[6] Ye, C. (2014) Wearable Devices-Make Technology besides You. Finance Technology Era, 4, 22-27.

[7] Wang, Y. (2014) Wearable Devices: Ignition Has Been Ignited. China Technolgy Fortune, 4, 52-55. 
[8] Xu, C. (2014) Coming into Fantastic Wireless Era. Communication World, 9, 16-17.

[9] Paro, J.A., Nazareli, R., Gurjala, A., Berger, A. and Lee, G.K. (2015) Video-Based Self-Review: Comparing Google Glass and GoPro Technologies. Annals of Plastic Surgery, 74, S71-S74. https://doi.org/10.1097/SAP.0000000000000423

[10] Alahakone, A.U., Senanayake, S.A. and Senanayake, C.M. (2010) Smart Wearable Device for Real Time Gait Event Detection during Running. 2010 IEEE Asia Pacific Conference on Circuits and Systems (APCCAS), Kuala Lumpur, 6-9 December 2010, 612-615. https://doi.org/10.1109/APCCAS.2010.5774975

[11] Koo, H.S. and Fallon, K. (2017) Preferences in Tracking Dimensions for Wearable Technology. International Journal of Clothing Science and Technology, 29, 180-199. https://doi.org/10.1108/IJCST-03-2016-0021

[12] Belsi, A., Papi, E. and McGregor, A.H. (2016) Impact of Wearable Technology on Psychosocial Factors of Osteoarthritis Management: A Qualitative Study. BMJ Open, 6, Article ID: e010064. https://doi.org/10.1136/bmjopen-2015-010064

[13] Waters, J.K. (2014) Could the Oculus Rift Redeem Virtual Reality in Higher Ed? https://campustechnology.com/articles/2014/10/09/could-the-oculus-rift-redeem-vi rtual-reality-in-higher-ed.aspx

[14] Turner, J. (2013) Other Amazing Uses for the Oculus Rift. https://www.ruhanirabin.com/other-amazing-uses-for-the-oculus-rift/

[15] Abrosimova, K. (2014) 5 Ways Virtual Reality Will Change Education. http://www.hypergridbusiness.com/2014/09/5-ways-virtual-reality-will-change-edu cation/

[16] Zhang, L. (2015) Review of Wearable Device Technology. Communication World, $15,250$.

[17] Neuger, B. (2015) Westonka Schools Lead in Smart PE. https://www.hometownsource.com/laker_pioneer/2015/12/17/westonka-schools-lea d-in-smart-pe/

[18] Chayka, K. (2012) Memoto Makes Lifecasting Way Easier Than Instagram. http://hyperallergic.com/59088/memoto-makes-lifecasting-way-easier-than-instagr $\underline{\mathrm{am}}$

[19] Kullman, J. (2013) New Monitoring Device to Protect Workers in Hazardous Conditions. https://asunews.asu.edu/20130904-labelle-exposure-track 